\title{
PROPERTIES OF CERTAIN PARTIAL DYNAMIC INTEGRODIFFERENTIAL EQUATIONS
}

\author{
DEEPAK B. PACHPATTE
}

\begin{abstract}
The aim of the present paper is to study the existence, uniqueness and some other properties of solutions of a certain partial dynamic integrodifferential equations. The Banach fixed point theorem and certain fundamental inequality with explicit estimates are used to establish our results.
\end{abstract}

\section{INTRODUCTION}

The study of time scale calculus was initiated by Stefan Hilger in his Ph.D dissertation which unifies the continuous and discrete calculus [4]. Since then many authors have worked on various aspects dynamic equations on timescale calculus [5, 6, 7, 8, 9, 9. Basic information on time scale calculus can be found in [1, 2, 3, 4. Many authors have studied various types of partial dynamic equations on time scales[7, 8, 10, 11, 14].In [12, 13, 15] have studied the integrodifferential equations and its properties. Motivated by the results in the above papers in this paper we study properties of certain partial dynamic integrodifferential equations. In what follows $\mathbb{R}$ denotes the set of real numbers and $\mathbb{T}$ denotes the arbitrary time scales. Now we give some basic definitions of time scale calculus. The function $f: \mathbb{T} \rightarrow \mathbb{R}$ is said to be rd-continuous if $f$ is continuous at each right dense point of $\mathbb{T}$ and is denoted by $C_{r d}$. Let two time scales with at least two point be denoted by $\mathbb{T}_{1}$ and $\mathbb{T}_{2}$ and $\Omega=\mathbb{T}_{1} \times \mathbb{T}_{2}$. The delta partial derivative of a real valued function $f$ on $\mathbb{T}_{1} \times \mathbb{T}_{2}$ has a $\Delta_{1}$ partial derivative $f^{\Delta_{1}}\left(t_{1}, t_{2}\right)$ with respect to $t_{1}$ if for each $\epsilon>0$ there exists a neighbourhood $U_{t_{1}}$ of $t_{1}$ such that.

$$
\left|f\left(\sigma_{1}\left(t_{1}\right), t_{2}\right)-f\left(s, t_{2}\right)-f^{\Delta_{1}}\left(t_{1}, t_{2}\right)\left(\sigma_{1}\left(t_{1}\right)-s\right)\right| \leq \varepsilon\left|\sigma_{1}\left(t_{1}\right)-s\right|
$$

for all $s \in U_{t_{2}}$. The delta partial derivative of a real valued function $f$ on $\mathbb{T}_{1} \times \mathbb{T}_{2}$ has a $\Delta_{2}$ partial derivative $f^{\Delta_{1}}\left(t_{1}, t_{2}\right)$ with respect to $t_{2}$ if for each $\eta>0$ there exists a neighbourhood $U_{t_{2}}$ of $t_{2}$ such that

$$
\left|f\left(t_{1}, \sigma_{2}\left(t_{2}\right)\right)-f\left(t_{1}, l\right)-f^{\Delta_{2}}\left(t_{1}, t_{2}\right)\left(\sigma_{2}\left(t_{2}\right)-l\right)\right| \leq \eta\left|\sigma_{2}\left(t_{2}\right)-l\right|
$$

2010 Mathematics Subject Classification. 26E70, 34N05, 26D10.

Key words and phrases. Banach fixed point theorem, Existence and Uniqueness, integral inequality,integrodifferential equations, time scales. 
for all $u \in U_{t_{1}}$.

The partial derivative of $w(x, y)$ for $(x, y) \in \Omega$ with respect to $x, y$ and $x y$ is denoted by $w^{\Delta_{1}}(x, y), w^{\Delta_{2}}(x, y)$ and $w^{\Delta_{1} \Delta_{2}}(x, y)=$ $w^{\Delta_{2} \Delta_{1}}(x, y)$. Suppose $I=[a, b]$ with $a<b$ and $\bar{\Omega}=\Omega \times I$. The partial derivative of $u(x, y, z)$ for $(x, y, z) \in C_{r d}(\bar{\Omega}, R)$ with respect to $x, y$ and $x y$ is defined by $w^{\Delta_{1}}(x, y, z), w^{\Delta_{2}}(x, y, z)$ and $w^{\Delta_{1} \Delta_{2}}(x, y, z)=$ $w^{\Delta_{2} \Delta_{1}}(x, y, z)$

In this paper we study the partial dynamic integrodifferential equation of the form

$u^{\Delta_{2} \Delta_{1}}(x, y, z)=F\left(x, y, z, u(x, y, z), u^{\Delta_{1}}(x, y, z), u^{\Delta_{2}}(x, y, z),(H u)(x, y, z)\right)$,

with the conditions

$$
u\left(x, y_{0}, z\right)=\alpha(x, z), u\left(x_{0}, y, z\right)=\beta(y, z)
$$

for $(x, y) \in \Omega$ where

$(H u)(x, y, z)=\int_{a}^{b} G\left(x, y, z, q, u(x, y, q), u^{\Delta_{1}}(x, y, q), u^{\Delta_{2}}(x, y, q)\right) \Delta q$,

where $G \in C_{r d}\left(\bar{\Omega} \times \mathbb{R}^{3}, \mathbb{R}\right), F \in C_{r d}\left(\bar{\Omega} \times \mathbb{R}^{4}, \mathbb{R}\right)$ and $\alpha, \beta \in C_{r d}\left(\mathbb{R}_{+} \times I, \mathbb{R}\right)$.

We have $u\left(x_{0}, y_{0}, z\right)=\alpha\left(x_{0}, z\right)=\beta\left(x_{0}, z\right)$.

Now for $u, u^{\Delta_{1}}, u^{\Delta_{2}} \in C_{r d}(\bar{\Omega}, \mathbb{R})$, we denote

$$
|u(x, y, z)|_{W}=|u(x, y, z)|+\left|u^{\Delta_{1}}(x, y, z)\right|+\left|u^{\Delta_{2}}(x, y, z)\right| .
$$

Let $S$ be the space function satisfying the condition

$$
|u(x, y, z)|_{W}=O\left(e_{\lambda}(x, y,|z|)\right),
$$

where $\lambda>0$ is a positive constant. In space $S$ we define norm $u$ by

$$
|u|_{s}=\sup _{(x, y, z) \in \Omega \times I}\left[|u(x, y, z)|_{w} e_{\Theta \lambda}(x, y,|z|)\right] .
$$

The norm defined (1.6) is clearly a Banach Space.

Then (1.5) implies that there is a constant $N \geq 0$ such that

$$
|u(x, y, z)|_{w} \leq N\left(e_{\lambda}(x, y,|z|)\right)
$$

and we have

$$
|u|_{s} \leq N
$$

The solution of (1.1) and (1.2) is a function $u(x, y, z) \in C_{r d}\left(\bar{\Omega}, \mathbb{R}^{n}\right)$ satisfying (1.1) and (1.2). It is easy to see that $u(x, y, z)$ with (1.1) and 
(1.2) satisfy the following dynamic integrodifferential equation.

$$
\begin{aligned}
& u(x, y, z) \\
& =\alpha(x, z)+\beta(y, z)-\alpha(0, z) \\
& +\int_{x_{0}}^{x} \int_{y_{0}}^{y} F\left(s, t, z, u(s, t, z), u^{\Delta_{1}}(s, t, z), u^{\Delta_{2}}(s, t, z),(H u)(s, t, z)\right) \Delta t \Delta s,
\end{aligned}
$$

$$
\begin{aligned}
\text { for } & (x, y, z) \in C_{r d}(\bar{\Omega}, \mathbb{R}) \\
& u^{\Delta_{1}}(x, y, z) \\
= & \alpha^{\Delta_{1}}(x, z) \\
& +\int_{y_{0}}^{y} F\left(x, t, z, u(x, t, z), u^{\Delta_{1}}(x, t, z), u^{\Delta_{2}}(x, t, z),(H u)(x, t, z)\right) \Delta t \\
& u^{\Delta_{2}}(x, y, z) \\
= & \beta^{\Delta_{2}}(y, z) \\
+ & \int_{x_{0}}^{x} F\left(s, y, z, u(x, t, z), u^{\Delta_{1}}(s, y, z), u^{\Delta_{2}}(s, y, z),(H u)(s, y, z)\right) \Delta s .
\end{aligned}
$$

We need following Lemma given in 3 .

Lemma [[3], Theorem 2.6] Let $u \in C_{r d}\left(\mathbb{T}, \mathbb{R}_{+}\right), a \in \mathbb{R}_{+}$

$$
u^{\Delta}(t) \leq a(t) u(t),
$$

for all $t \in \mathbb{T}^{k}$, then

for all $t \in \mathbb{T}^{k}$.

$$
u(t) \leq u\left(t_{0}\right) e_{a}\left(t, t_{0}\right)
$$

\section{MAin Results}

Now we give our main results

Theorem 1.1 Suppose that the functions $F, G$ in (1.1) satisfy the condition

$$
\begin{gathered}
\left|F\left(x, y, z, u_{1}, u_{2}, u_{3}, u_{4}\right)-F\left(x, y, z, \overline{u_{1}}, \overline{u_{2}}, \overline{u_{3}}, \overline{u_{4}}\right)\right| \\
\leq M(x, y, z)\left[\left|u_{1}-\overline{u_{1}}\right|+\left|u_{2}-\overline{u_{2}}\right|+\left|u_{3}-\overline{u_{3}}\right|+\left|u_{4}-\overline{u_{4}}\right|\right], \\
\left|G\left(x, y, z, q, u_{1}, u_{2}, u_{3}\right)-G\left(x, y, z, q, \overline{u_{1}}, \overline{u_{2}}, \overline{u_{3}}\right)\right|
\end{gathered}
$$




$$
\leq K(x, y, z, q)\left[\left|u_{1}-\overline{u_{1}}\right|+\left|u_{2}-\overline{u_{2}}\right|+\left|u_{3}-\overline{u_{3}}\right|\right],
$$

where $M \in C_{r d}\left(\bar{\Omega}, \mathbb{R}_{+}\right)$and $K \in C_{r d}\left(\bar{\Omega} \times I, \mathbb{R}_{+}\right)$.

For $\lambda$ as in (1.5), there exists a nonnegative $\gamma_{i}(i=1,2,3)$ such that

$$
\begin{aligned}
& \int_{x_{0}}^{x} \int_{y_{0}}^{y} M(s, t, z)\left[e_{\lambda}(s, t,|z|)\right. \\
& \left.+\int_{a}^{b} k(s, t, z, q) e_{\lambda}(s, t,|q|) \Delta q\right] \Delta t \Delta s \leq \gamma_{1} e_{\lambda}(x, y,|z|), \\
& \int_{y_{0}}^{y} M(x, t, z)\left[e_{\lambda}(x, t,|q|)\right. \\
& \left.+\int_{a}^{b} k(x, t, z, q) e_{\lambda}(x, t,|q|) \Delta q\right] \Delta t \leq \gamma_{2} e_{\lambda}(x, y,|z|), \\
& \int_{x_{0}}^{x} M(s, y, z)\left[e_{\lambda}(x, t,|z|)\right. \\
& \left.+\int_{a}^{b} k(s, y, z, q) e_{\lambda}(s, y,|q|) \Delta q\right] \Delta s \leq \gamma_{3} e_{\lambda}(x, y,|z|),
\end{aligned}
$$

for $x, y \in \Omega, z \in I$.

There exist nonnegative constants $\eta_{i}(i=1,2,3)$ such that

$$
\begin{aligned}
& |\alpha(x, z)|+|\beta(y, z)|+|\alpha(0, z)| \\
& +\int_{x_{0}}^{x} \int_{y_{0}}^{y}|f(s, t, z, 0,0,(H 0)(s, t, z))| \Delta t \Delta s \leq \eta_{1} e_{\lambda}(x, y,|z|) \\
& \left|\alpha^{\Delta}(x, z)\right|+\int_{y_{0}}^{y}|F(x, t, z, 0,0,0,(H 0)(x, t, z))| \Delta t \leq \eta_{2} e_{\lambda}(x, y,|z|) \\
& \left|\beta^{\Delta}(y, z)\right|+\int_{y_{0}}^{y}|F(s, y, z, 0,0,0,(H 0)(s, y, z))| \Delta s \leq \eta_{3} e_{\lambda}(x, y,|z|)
\end{aligned}
$$


where $\alpha, \beta$ are as in (1.2).

If $\gamma=\gamma_{1}+\gamma_{2}+\gamma_{3}<1$ then problem (1.1) - (1.2) has a unique solution $u(x, y, z)$ on $(1.1)-(1.2)$ in $S$.

Proof. Let $u(x, y, z) \in S$ and define the operator $\mathbb{T}$ by

$(T u)(x, y, z)$

$=\alpha(x, z)+\beta(y, z)-\alpha(0, z)$

$+\int_{x_{0}}^{x} \int_{y_{0}}^{y} F\left(s, t, z, u(s, t, z), u^{\Delta_{1}}(s, t, z), u^{\Delta_{2}}(s, t, z),(H u)(s, t, z)\right) \Delta t \Delta s$.

Now we show that $P$ maps $S$ into itself. $T u$ is rd-continuous on $\Omega \times I$ and $T u \in R$.

From (2.9) and given hypotheses we have

$$
\begin{aligned}
& (P u)(x, y, z) \\
& \leq|\alpha(x, z)|+|\beta(y, z)|+|\alpha(0, z)| \\
& +\int_{x_{0}}^{x} \int_{y_{0}}^{y} \mid F\left(s, t, z, u(s, t, z), u^{\Delta_{1}}(s, t, z), u^{\Delta_{2}}(s, t, z),(H u)(s, t, z)\right) \\
& -F(s, t, z, 0,0,0,(H 0)(s, t, z)) \mid \Delta t \Delta s \\
& +\int_{x_{0}}^{x} \int_{y_{0}}^{y}|F(s, t, z, 0,0,0,(H 0)(s, t, z))| \Delta t \Delta s \\
& \leq \eta_{1} e_{\lambda}(x, y,|z|)+\int_{x_{0}}^{x} \int_{y_{0}}^{y} M(s, t, z)\left[e_{\lambda}(s, t,|z|)|u(s, t, z)| e_{\Theta \lambda}(s, t,|z|)\right. \\
& \left.+\int_{a}^{b} k(x, y, z, q) e_{\lambda}(s, t,|q|)|u(x, y,|q|)|_{W} e_{\Theta \lambda}(s, t,|q|) \Delta q\right] \Delta t \Delta s \\
& \leq \eta_{1} e_{\lambda}(x, y,|z|)+|u|_{s} \int_{x_{0}}^{x} \int_{y_{0}}^{y} M(s, t, z)\left[e_{\lambda}(s, t,|z|)\right. \\
& \left.+\int_{a}^{b} k(x, y, z, q) e_{\lambda}(s, t,|q|) \Delta q\right] \Delta t \Delta s \\
& \leq\left[\eta_{1}+N \gamma_{1}\right] e_{\lambda}(x, y,|z|) \text {. }
\end{aligned}
$$


Delta differentiating on both sides of (2.9) with respect to $x$ and (1.8) we have

$$
\begin{aligned}
& \left|(P u)^{\Delta_{1}}(x, y, z)\right| \\
& \leq \alpha^{\Delta_{1}}(x, z) \\
& +\int_{y_{0}}^{y} \mid F\left(x, t, z, u(x, t, z), u^{\Delta_{1}}(x, t, z), u^{\Delta_{2}}(x, t, z),(H u)(x, t, z)\right) \\
& -F(x, t, z, 0,0,0,(H 0)(x, t, z)) \mid \Delta t \\
& +\int_{y_{0}}^{y}|F(x, t, z, 0,0,0,(H 0)(x, t, z))| \Delta t \\
& \leq \eta_{2} e_{\lambda}(x, y,|z|)+|u|_{s} \int_{y_{0}}^{y} M(x, t, z)\left[e_{\lambda}(x, t,|z|)\right. \\
& \left.+\int_{a}^{b} k(x, t, z, q) e_{\lambda}(x, t,|q|) \Delta q\right] \Delta t \\
& \leq\left[\eta_{2}+N \gamma_{2}\right] e_{\lambda}(x, y,|z|) .
\end{aligned}
$$

Similarly we have

$$
\left|(P u)^{\Delta_{2}}(x, y, z)\right| \leq\left[\eta_{3}+N \gamma_{3}\right] e_{\lambda}(x, y,|z|) .
$$

From $(2.10)-(2.12)$ we have

$$
|P u|_{s} \leq\left[\left(\eta_{1}+\eta_{2}+\eta_{3}\right)+N \gamma\right] .
$$

Thus proving that $P$ maps $S$ into itself.

Now we show that operator $P$ is a contraction map. Let $u(x, y, z), \bar{u}(x, y, z) \in$ $S$. From (2.9) we have

$$
\begin{aligned}
& |(P u)(x, y, z)-(P \bar{u})(x, y, z)| \\
& \leq \int_{x_{0}}^{x} \int_{y_{0}}^{y} \mid F\left(s, t, z, u(s, t, z), u^{\Delta_{1}}(s, t, z), u^{\Delta_{2}}(s, t, z),(H u)(s, t, z)\right) \\
& -F\left(s, t, z, \bar{u}(s, t, z), \bar{u}^{\Delta_{1}}(s, t, z), \bar{u}^{\Delta_{2}}(s, t, z),(H \bar{u})(s, t, z)\right) \mid \Delta t \Delta s \\
& \leq|u-\bar{u}|_{s} \int_{x_{0}}^{x} \int_{y_{0}}^{y} M(x, t, z)\left[e_{\lambda}(s, t,|z|)\right.
\end{aligned}
$$




$$
\begin{aligned}
& \left.+\int_{a}^{b} k(s, t, z, q) e_{\lambda}(s, t,|q|) \Delta q\right] \Delta t \Delta s \\
& \leq|u-\bar{u}|_{s} \gamma_{1} e_{\lambda}(x, y,|z|) .
\end{aligned}
$$

Similarly delta differentiating both sides of (2.12) with respect to $x$ and $y$ we have

$$
\left|(P u)^{\Delta_{1}}(x, y, z)-(P \bar{u})^{\Delta_{1}}(x, y, z)\right| \leq|u-\bar{u}|_{s} \gamma_{2} e_{\lambda}(x, y,|z|),
$$

and

$$
\left|(P u)^{\Delta_{2}}(x, y, z)-(P \bar{u})^{\Delta_{2}}(x, y, z)\right| \leq|u-\bar{u}|_{s} \gamma_{3} e_{\lambda}(x, y,|z|) .
$$

From $(2.13)-(2.15)$ we obtain

$$
|P u-P \bar{u}|_{s} \leq \gamma|u-\bar{u}|_{s} .
$$

Since $\gamma<1, P$ has a unique fixed point in $S$ by Banach fixed point theorem. The fixed point of $P$ is a solution of $(1.1)-(1.2)$. This completes the proof.

\section{Properties of SOlutions}

Now we study the properties of solution of dynamic integrodifferential equation of the form

$$
u^{\Delta_{2} \Delta_{1}}(x, y, z)=f(x, y, z, u(x, y, z),(h u)(x, y, z)),
$$

with (1.2) for $(x, y, z) \in \bar{\Omega}$ where

$$
(h u)(x, y, z)=\int_{a}^{b} j(x, y, z, q, u(x, y, q)) d q,
$$

in which $i \in C_{r d}(\bar{\Omega} \times \mathbb{R}, \mathbb{R}), f \in C_{r d}\left(\Omega \times \mathbb{R}^{2}, \mathbb{R}\right)$.

Now we prove the following dynamic inequality which can be used in studying some properties of solutions.

Theorem 3.1 Let $w, p \in C_{r d}\left(\bar{\Omega}, \mathbb{R}_{+}\right), \in C_{r d}\left(\bar{\Omega} \times I, \mathbb{R}_{+}\right)$and $c \geq 0$ a constant. If

$$
\begin{aligned}
w(x, y, z) \leq & c+\int_{x_{0}}^{x} \int_{y_{0}}^{y}[p(s, t, z) w(s, t, z) \\
& \left.+\int_{a}^{b} r(s, t, z, q) w(s, t, q) \Delta q\right] \Delta t \Delta s,
\end{aligned}
$$


for $(x, y, z) \in \Omega$ then

$$
w(x, y, z) \leq c e_{Q(x, y, z)}\left(x, x_{0}\right),
$$

where $(x, y, z) \in \bar{\Omega}$ and

$$
Q(x, y, z)=\int_{y_{0}}^{y}\left[p(s, t, Z) \int_{a}^{b} r(s, t, Z, q) \Delta q\right] \Delta s .
$$

Proof. For an arbitrary $Z \in I$ from (3.3) we have

$$
\begin{aligned}
w(x, y, Z) \leq c+ & \int_{x_{0}}^{x} \int_{y_{0}}^{y}[p(s, t, Z) w(s, t, Z) \\
& \left.+\int_{a}^{b} r(s, t, Z, q) w(s, t, q) \Delta q\right] \Delta t \Delta s .
\end{aligned}
$$

Put

$$
m(s, t)=p(s, t, Z) w(s, t, Z)+\int_{a}^{b} r(s, t, z, q) w(s, t, q) \Delta q .
$$

The inequality (3.6) becomes

$$
w(x, y, Z) \leq c+\int_{x_{0}}^{x} \int_{y_{0}}^{y} m(s, t) \Delta t \Delta s
$$

Now define

$$
v(x, y)=c+\int_{x_{0}}^{x} \int_{y_{0}}^{y} m(s, t) \Delta t \Delta s
$$

then

$$
v(0, y)=v(x, 0)=c, w(x, y, Z) \leq v(x, y) .
$$

Delta differentiating both sides of (3.9) with respect to $x$ and $y$ using (3.7) and (3.10) we have

$$
\begin{aligned}
v^{\Delta_{2} \Delta_{1}}(x, y) & =m(x, y) \\
& =p(x, y, Z) w(x, y, Z)+\int_{a}^{b} r(x, y, Z, q) w(x, y, q) \Delta q
\end{aligned}
$$




$$
\leq v(x, y)\left[p(x, y, Z)+\int_{a}^{b} r(s, t, Z, q) \Delta q\right] .
$$

By keeping $x$ fixed in (3.11), and taking $y=t$ and delta integrating with respect to second variable from $y_{0}$ to $y$. Using the fact that

$$
\begin{aligned}
v^{\Delta_{1}}(x, y) & \leq \int_{y_{0}}^{y}\left[p(x, t, Z)+\int_{a}^{b} r(x, t, Z, q) \Delta q\right] v(x, t) \Delta t \\
& \leq v(x, y) \int_{y_{0}}^{y}\left[p(x, t, Z)+\int_{a}^{b} r(x, t, Z, q) \Delta q\right] \Delta t \\
& \leq v(x, y) Q(x, y, Z) .
\end{aligned}
$$

Now treating $y$ fixed in (3.12) and applying Lemma we have

$$
v(x, y) \leq c e_{Q(x, y, Z)}\left(x, x_{0}\right) .
$$

Because $Z$ is arbitrary and using (3.10) we get (3.9).

Theorem 3.2 Suppose the functions $f, j$ in (3.1),(3.2) satisfy the conditions

$$
\begin{gathered}
|f(x, y, z, u, v)-f(x, y, z, \bar{u}, \bar{v})| \leq p_{1}(x, y, z)[|u-\bar{u}|+|v-\bar{v}|] \\
|j(x, y, z, q, u)-j(x, y, z, \bar{q}, \bar{u})| \leq p_{2}(x, y, z, q)|u-\bar{u}|
\end{gathered}
$$

where $p_{1} \in C_{r d}\left(\bar{\Omega}, \mathbb{R}_{+}\right), p_{2} \in C_{r d}\left(\bar{\Omega} \times I, \mathbb{R}_{+}\right), c \geq 0$ and

$$
\int_{x_{0}}^{x} \int_{y_{0}}^{y}\left[p_{1}(s, t, z)+\int_{a}^{b} p_{1}(s, t, z, q) \Delta q\right] \Delta t \Delta s<\infty,
$$

then the problem $(3.1)-(1.1)$ has at most one solution.

Proof. Let $u_{1}(x, y, z)$ and $u_{2}(x, y, z)$ be two solutions of problem $(3.1)-(1.1)$.

$$
\begin{aligned}
& \left|u_{1}(x, y, z)-u_{2}(x, y, z)\right| \\
& \leq \int_{x_{0}}^{x} \int_{y_{0}}^{y} \mid f\left(s, t, z, u_{1}(s, t, z),\left(h u_{1}\right)(s, t, z)\right) \\
& -f\left(s, t, z, u_{2}(s, t, z),\left(h u_{2}\right)(s, t, z)\right) \mid \Delta t \Delta s \\
& \leq \int_{x_{0}}^{x} \int_{y_{0}}^{y}\left[p_{1}(s, t, z)\left|u_{1}(s, t, z)-u_{2}(s, t, z)\right|\right.
\end{aligned}
$$




$$
\begin{aligned}
& \left.+\left|\left(h u_{1}\right)(s, t, z)-\left(h u_{2}\right)(s, t, z)\right|\right] \Delta t \Delta s \\
& \leq \int_{x_{0}}^{x} \int_{y_{0}}^{y}\left[p_{1}(s, t, z)\left|u_{1}(s, t, z)-u_{2}(s, t, z)\right|\right. \\
& \left.+\int_{a}^{b} p_{1}(s, t, z, q)\left|u_{1}(s, t, q)-u_{2}(s, t, q)\right| \Delta q\right] \Delta t \Delta s .
\end{aligned}
$$

Now applying Theorem 3.1to (3.17) yields $\left|u_{1}(x, y, z)-u_{2}(x, y, z)\right| \leq 0$ which gives $u_{1}(x, y, z)=u_{2}(x, y, z)$. This proves that there is at most one solution to problem $(3.1)-(1.1)$.

Now we prove the theorem which gives the boundedness of solution of $(3.1)-(1.1)$.

Theorem 3.3. Suppose the function $f, j, \alpha, \beta$ in $(3.1)-(1.1)$ satisfy the conditions

$$
\begin{gathered}
|f(x, y, z, u, v)| \leq p_{1}(x, y, z)[|u|+|v|], \\
|j(x, y, z, u, v)| \leq p_{2}(x, y, z, q)|u|, \\
|\alpha(x, z)+\beta(y, z)-\alpha(0, z)| \leq c,
\end{gathered}
$$

where $p_{1} \in C_{r d}\left(\Omega, \mathbb{R}_{+}\right), p_{2} \in C_{r d}\left(\Omega \times I, \mathbb{R}_{+}\right), c \geq 0$ is a constant and the condition (3.16) holds. Then solution $u(x, y, z)$ is bounded and

$$
|u(x, y, z)| \leq c e_{Q(x, y, z)}\left(x, x_{0}\right),
$$

for $(x, y, z) \in \bar{\Omega}$

Proof. Since $u(x, y, z)$ is a solution of $(3.1)-(1.1)$. We have

$$
\begin{aligned}
|u(x, y, z)| & \leq|\alpha(x, z)+\beta(y, z)-\alpha(0, z)| \\
& +\int_{x_{0}}^{x} \int_{y_{0}}^{y}|f(s, t, z, u(s, t, z),(h u)(s, t, z))| \Delta t \Delta s \\
& \leq c+\int_{x_{0}}^{x} \int_{y_{0}}^{y}\left[p_{1}(s, t, z)|u(s, t, z)|\right. \\
& \left.+\int_{a}^{b} p_{2}(s, t, z, q)|u(s, t, q)| \Delta q\right] \Delta t \Delta s .
\end{aligned}
$$

Now an application of Theorem 3.1 to (3.22) yields (3.21) thus proving the boundedness of solution.

Now we give the dependency of solution of equation on given condition 
Theorem 3.4. Suppose the function $f, k$ in (3.1), (3.2) satisfy the conditions (3.14), (3.15) and the condition (3.16) holds. Let $u(x, y, z)$ and $v(x, y, z)$ be the solutions of equation with condition (1.2) and

$$
v(x, 0, z)=\bar{\alpha}(x, z), v(0, y, z)=\bar{\beta}(y, z),
$$

respectively and

$$
|\alpha(x, z)+\beta(y, z)-\alpha(0, z)-[\bar{\alpha}(x, z)+\bar{\beta}(y, z)-\bar{\alpha}(0, z)]| \leq a,
$$

where $\alpha, \beta, \bar{\alpha}, \bar{\beta} \in C_{r d}\left(\mathbb{R}_{+} \times I, \mathbb{R}\right)$ and $a \geq 0$ is constant. Then

$$
|u(x, y, z)-v(x, y, z)| \leq a e_{Q(x, y, z)}\left(x, x_{0}\right) .
$$

Proof. Since $u(x, y, z)$ and $v(x, y, z)$ are solutions of (3.1)-(1.1) and (3.1) - (3.23) and the given conditions we have

$$
\begin{aligned}
& |u(x, y, z)-u(x, y, z)| \\
& \leq \mid \alpha(x, z)+\beta(y, z)-\alpha(0, z) \\
& -[\bar{\alpha}(x, z)+\bar{\beta}(y, z)-\bar{\alpha}(0, z)] \mid \\
& +\int_{x_{0}}^{x} \int_{y_{0}}^{y} \mid f(s, t, z, u(s, t, z),(h u)(s, t, z)) \\
& -f(s, t, z, v(s, t, z),(h u)(s, t, z)) \mid \Delta t \Delta s \\
& \leq a+\int_{x_{0}}^{y} \int_{y_{0}}^{y}\left[p_{1}(s, t, z)|u(s, t, z)-v(s, t, z)|\right. \\
& \left.+\int_{a}^{b} p_{2}(s, t, z, q)|u(s, t, z)-v(s, t, z)| \Delta q\right] \Delta t \Delta s .
\end{aligned}
$$

Now an application of Theorem 3.1 to (3.26) gives the estimate (3.25) which gives the dependency of solution of equation (3.1) on given conditions.

Acknowledgement. This research is supported by Science and Engineering Research Board(SERB), New Delhi, India, Sanct. No. SB/S4/MS:861/13

\section{References}

\section{REFERENCES}

[1] M. Bohner and A. Peterson, Dynamic equations on time scales, Birkhauser Boston/Berlin, (2001).

[2] M. Bohner and A. Peterson, Advances in Dynamic equations on time scales, Birkhauser Boston/Berlin, (2003). 
[3] E.A.Bohner, M. Bohner and F. Akin, Pachpatte inequalities on on time scales, J. Inequal. Pure Appl. Math.,,6(1)(2005), Art. 6.

[4] S. Hilger, Analysis on Measure chain-A unified approch to continuous and discrete calculus, Results. Math., 18:18-56, 1990.

[5] D. B. Pachpatte, Explicit estimates on integral inequalities with time scale, J. Inequal. Pure. Appl. Math., Vol. 7, Issue 4, Artivle 143, 2006.

[6] D. B. Pachpatte, Integral Inequalitys for partial dynamic equations on time scales, Electron. J. Differential Equations, Vol. 2012 (2012), No. 50, 1-7.

[7] D. B. Pachpatte, Properties of solutions to nonlinear dynamic integral equations on Time Scales, Electron. J. Differential Equations, Vol. 2008(2008). No. 130. pp.1-8.

[8] D. B. Pachpatte, Properties of some partial dynamic equations on time scales, International Journal of Partial Differential Equations, Vol. 2013, Art. ID 345697, 9 pages

[9] D. B. Pachpatte, Properties of some dynamic Integral equations on time scales, Ann. Funct. Anal. , Vol.4, No2.,2013.

[10] B. Jackson, Partial dyamic equations on time scales, J. Comput. Appl. Math. ,Vol.186, Issue 2, Feb 2006, p. 391-415.

[11] Y. Suna, T. Hassanb, Some nonlinear dynamic integral inequalities on time scales, Appl. Math. Comput., Vol 220, 2013, P. 221-225.

[12] G. Liu, X. Xiang, Y. Peng Nonlinear integrodifferential equations and optimal control problems on time scales, Comput. Math. Appl., Vol 61,Issue 2, Jan 2011, P. 115-169.

[13] A. S. Nowak, Integrodifferential equations on time scales with HenstockKurzweil-pettis Delta Integrals, Abstr. Appl. Anal., Vol 2010,Art. ID 836347, p. 17.

[14] F. Menga, J. Shaoa, Some new Volterra Fredholm type dynamic integral inequalities on time scales, Appl. Math. Comput., Vol. 223, 2013, p. 444-451.

[15] Y. Xing, M. Han, G. Zheng Initial value problem for first order integrodifferential equation of Voltera type on Time scales, Nonlinear Anal., Vol. 60, Issue 3,Feb 2005, p. 429-442.

Deepak B. Pachpatte

Department of Mathematics, Dr. Babasaheb Ambedkar Marathwada

University, Aurangabad, Maharashtra 431004, India

E-mail address: pachpatte@gmail.com 